This item was submitted to Loughborough's Research Repository by the author.

Items in Figshare are protected by copyright, with all rights reserved, unless otherwise indicated.

\title{
Consistent weight restrictions in data envelopment analysis
}

PLEASE CITE THE PUBLISHED VERSION

http://dx.doi.org/10.1016/j.ejor.2015.01.037

\section{PUBLISHER}

(c) Elsevier

\section{VERSION}

AM (Accepted Manuscript)

\section{PUBLISHER STATEMENT}

This work is made available according to the conditions of the Creative Commons Attribution-NonCommercialNoDerivatives 4.0 International (CC BY-NC-ND 4.0) licence. Full details of this licence are available at: https://creativecommons.org/licenses/by-nc-nd/4.0/

\section{LICENCE}

CC BY-NC-ND 4.0

\section{REPOSITORY RECORD}

Podinovski, Victor V., and Tatiana Bouzdine-Chameeva. 2019. "Consistent Weight Restrictions in Data Envelopment Analysis". figshare. https://hdl.handle.net/2134/18171. 


\title{
Consistent weight restrictions in data envelopment analysis
}

\author{
Victor V. Podinovski ${ }^{\mathrm{a}, *}$, Tatiana Bouzdine-Chameeva ${ }^{\mathrm{b}}$ \\ ${ }^{a}$ Warwick Business School, University of Warwick, Coventry CV4 7AL, UK \\ ${ }^{b}$ KEDGE Business School, 680 cours de la Libération, 33405 Talence Cedex, France
}

\begin{abstract}
It has recently been shown that the incorporation of weight restrictions in models of data envelopment analysis (DEA) may induce free or unlimited production of output vectors in the underlying production technology, which is expressly disallowed by standard production assumptions. This effect may either result in an infeasible multiplier model with weight restrictions or remain undetected by normal efficiency computations. The latter is potentially troubling because even if the efficiency scores appear unproblematic, they may still be assessed in an erroneous model of production technology. Two approaches to testing the existence of free and unlimited production have recently been developed: computational and analytical. While the latter is more straightforward than the former, its application is limited only to unlinked weight restrictions. In this paper we develop several new analytical conditions for a larger class of unlinked and linked weight restrictions.
\end{abstract}

Keywords: data envelopment analysis, weight restrictions, production trade-offs

\section{Introduction}

The constant and variable (CRS and VRS) models of data envelopment analysis (DEA) can be stated in the form of mutually dual envelopment and multiplier linear programs (Charnes et al. 1978, Banker et al. 1984, Cooper et al. 2007, Thanassoulis et al. 2008). The DEA envelopment programs are usually interpreted using the notion of production technology generated by the set of observed units. The DEA multiplier models employ the dual approach based on the use of variable input and output weights.

It is well-known that the incorporation of additional weight restrictions in multiplier DEA models generally results in the expansion of the production technology (Roll et al. 1991, Allen et al. 1997). This shifts the production frontier away from the observed units and leads to their lower efficiency. Therefore, the use of weight restrictions generally improves the discrimination of DEA models on efficiency, which is considered desirable in many applications.

The use of weight restrictions may lead to the infeasibility of the multiplier models (Allen et al. 1997). In a recent paper, Podinovski and Bouzdine-Chameeva (2013) show that such an infeasibility translates into the existence of free or unlimited production of vectors of outputs in the production technology. They also show that even if the multiplier model is feasible for each observed unit under the assessment, the use of weight restrictions may still induce free or unlimited production, although this effect may be undetected by standard calculations.

\footnotetext{
*Corresponding author

Email addresses: victor.podinovski@wbs.ac.uk (Victor V. Podinovski), tatiana.chameeva@kedgebs.com (Tatiana Bouzdine-Chameeva)
} 
Free and unlimited production is expressly disallowed by established axiomatic characterizations of technology - see, e.g., Färe et al. (1985). The ability of weight restrictions to induce free or unlimited production in the underlying technology without being detected is troubling. Indeed, even if the results of efficiency computations with weight restrictions appear normal and unsuspicious, the model of technology may still violate one of the basic production assumptions.

In this paper we call weight restrictions consistent with the set of observed units if their incorporation in the multiplier DEA model does not create free and unlimited production. Otherwise, the weight restrictions are referred to as inconsistent.

Because inconsistent weight restrictions may be impossible to detect by standard calculations of efficiency, we need to have a method that allows us to verify if this problem occurs, so that the weight restrictions could be reassessed. Podinovski and Bouzdine-Chameeva (2013) develop two approaches for testing the existence of free and unlimited production.

The general computational approach requires solving either a single specially constructed envelopment model with the original data set or, alternatively, several standard envelopment models with an extended set of observed units.

The analytical approach requires verifying that there exist strictly positive input and output weights that satisfy the weight restrictions, or a slightly relaxed similar condition. However, unlike the general computational approach, this analytical condition applies only if the weight restrictions are unlinked, i.e., if the weight restrictions are specified separately for the input and output weights.

The purpose of this paper is to develop more general necessary and sufficient analytical conditions that extend to a larger class of unlinked and linked weight restrictions. These new conditions make it unnecessary to formulate, program and solve the nonstandard linear programs required by the above general computational approach. It should be noted that the new sufficient analytical conditions do not extend to the types of linked weight restrictions whose consistency depends on the particular set of observed units. The consistency of such types of weight restrictions may be verified by the existing computational approaches.

\section{Preliminaries}

Assume there are $N$ observed units $\left(X_{j}, Y_{j}\right)$, where $X_{j} \in \mathbb{R}_{+}^{m} \backslash\{0\}$ is the vector of inputs and $Y_{j} \in \mathbb{R}_{+}^{s} \backslash\{0\}$ is the vector of outputs, for all $j=1, \ldots, N$. Denote $\Omega$ the set of observed units (the data set).

The CRS and VRS multiplier DEA models are stated in terms of variable vectors of input and output weights $v \in \mathbb{R}_{+}^{m}$ and $u \in \mathbb{R}_{+}^{s}$. The use of additional weight restrictions in such models is a common technique that aims at reducing the flexibility of input and output weights (Allen et al. 1997). ${ }^{1}$

Below we consider the case of homogeneous weight restrictions ${ }^{2}$

$$
Q_{t}^{\top} u-P_{t}^{\top} v \leq 0, \quad t=1, \ldots, K
$$

\footnotetext{
${ }^{1}$ Our development concerns the standard CRS and VRS models. In particular, it is assumed that the inputs and outputs are nonnegative and satisfy the assumption of free disposability. As discussed in the concluding section, our results also extend, without any modification, to a variety of other DEA models that are based on the same standard CRS and VRS technologies and differ only in the way the units are projected on their boundaries. The extension of our results to DEA models based on technologies other than the standard CRS and VRS technologies requires further research that goes beyond the scope of this paper. In particular, this includes models with negative output values and technologies based on the assumption of weak disposability of bad outputs.

${ }^{2}$ Homogeneous weight restrictions, unlike, for example, absolute weight bounds, have constant 0 on the right-hand side of (1).
} 
In (1), $Q_{t} \in \mathbb{R}^{s}$ and $P_{t} \in \mathbb{R}^{m}$ are constant vectors whose component can be positive, negative or zero. If, for a particular $t$, both vectors $Q_{t}$ and $P_{t}$ are nonzero, the corresponding weight restriction in (1) is referred to as linked. Otherwise, if one of the two vectors is zero (or missing), the weight restriction is unlinked. ${ }^{3}$

Under the assumption of CRS and subject to weight restrictions (1), the output radial efficiency of the unit $\left(X_{o}, Y_{o}\right)$ is the inverse to the optimal value $\eta^{*}$ of the following multiplier model:

$$
\begin{aligned}
\eta^{*}=\min & X_{o}^{\top} v \\
\text { subject to } & Y_{o}^{\top} u=1, \\
& Y_{j}^{\top} u-X_{j}^{\top} v \leq 0, \quad j=1, \ldots, N, \\
& Q_{t}^{\top} u-P_{t}^{\top} v \leq 0, \quad t=1, \ldots, K, \\
& u, v \geq 0 .
\end{aligned}
$$

Similarly, the CRS input radial efficiency of the unit $\left(X_{o}, Y_{o}\right)$ is equal to the optimal value $\theta^{*}$ in the following program:

$$
\begin{aligned}
\theta^{*}=\max & Y_{o}^{\top} u \\
\text { subject to } & X_{o}^{\top} v=1, \text { and }(2.3)-(2.5) .
\end{aligned}
$$

Under the assumption of VRS, models (2) and (3) include an additional sign-free variable $u_{0}$ and require a straightforward modification. ${ }^{4}$

\section{Production trade-offs}

The effect of the incorporation of weight restrictions (1) in multiplier DEA models may be illustrated by observing the corresponding changes to their dual models. For example, the dual to model (2) is the output-oriented envelopment model

$$
\begin{array}{ll}
\eta^{*}=\max & \eta \\
\text { subject to } & \sum_{j=1}^{N} \lambda_{j} X_{j}+\sum_{t=1}^{K} \pi_{t} P_{t}+S_{X}=X_{o}, \\
& \sum_{j=1}^{N} \lambda_{j} Y_{j}+\sum_{t=1}^{K} \pi_{t} Q_{t}-S_{Y}=\eta Y_{o}, \\
& \lambda, \pi, S_{X}, S_{Y} \geq 0, \eta \text { sign free. }
\end{array}
$$

In the above program, $\lambda=\left(\lambda_{1}, \ldots, \lambda_{N}\right)$ is the intensity vector corresponding to inequalities $(2.3), \pi=\left(\pi_{1}, \ldots, \pi_{K}\right)$ is the vector of dual variables to inequalities $(2.4)$, and $S_{X} \in \mathbb{R}^{m}$ and $S_{Y} \in \mathbb{R}^{s}$ are the vectors of input and output slack variables, respectively.

\footnotetext{
${ }^{3}$ Unlinked weight restrictions are also referred to as assurance region of type I or polyhedral cone ratio (Charnes et al. 1989). Linked weight restrictions are assurance region of type II (Thompson et al. 1990).

${ }^{4}$ See, e.g., models (8) and (9) in Podinovski and Bouzdine-Chameeva (2013).
} 
Similarly, the dual to model (3) is the input-oriented envelopment model:

$$
\begin{array}{ll}
\theta^{*}=\min & \theta \\
\text { subject to } & \sum_{j=1}^{N} \lambda_{j} X_{j}+\sum_{t=1}^{K} \pi_{t} P_{t}+S_{X}=\theta X_{o}, \\
& \sum_{j=1}^{N} \lambda_{j} Y_{j}+\sum_{t=1}^{K} \pi_{t} Q_{t}-S_{Y}=Y_{o}, \\
& \lambda, \pi, S_{X}, S_{Y} \geq 0, \theta \text { sign free. }
\end{array}
$$

The first group of terms with variables $\lambda_{j}$ on the left-hand side of equalities (4.2), (4.3) and (5.2), (5.3) describes a composite unit in the standard CRS technology. This unit is further modified by the terms

$$
\left(P_{t}, Q_{t}\right), \quad t=1, \ldots, K
$$

used in proportions $\pi_{t} \geq 0$. Following Podinovski (2004), the terms (6) may be interpreted as production trade-offs. Such trade-offs describe simultaneous changes to the inputs and outputs of the units in the CRS technology and lead to its expansion. ${ }^{5}$

It is clear that both the output-oriented model (4) and input-oriented model (5) assess the efficiency of the unit $\left(X_{o}, Y_{o}\right)$ in the same CRS technology extended by production trade-offs (6) (or equivalent to them weight restrictions (1)). This technology is defined as follows. ${ }^{6}$

Definition 1. The CRS technology with production trade-offs (6), denoted $\mathcal{T}_{C R S-T O}$, is the set of all units $(X, Y) \in \mathbb{R}_{+}^{m} \times \mathbb{R}_{+}^{s}$ for which there exist intensity vectors $\lambda \in \mathbb{R}_{+}^{N}, \pi \in \mathbb{R}_{+}^{K}$, and slack vectors $S_{X} \in \mathbb{R}_{+}^{m}$ and $S_{Y} \in \mathbb{R}_{+}^{s}$ such that

$$
\begin{aligned}
& \sum_{j=1}^{N} \lambda_{j} X_{j}+\sum_{t=1}^{K} \pi_{t} P_{t}+S_{X}=X \\
& \sum_{j=1}^{N} \lambda_{j} Y_{j}+\sum_{t=1}^{K} \pi_{t} Q_{t}-S_{Y}=Y .
\end{aligned}
$$

Note that Definition 1 requires that the units of technology $\mathcal{T}_{C R S-T O}$ be nonnegative, and this condition is missing from both envelopment models (4) and (5). In the former model this does not cause any problems: because $\eta$ is maximized, the unit $\left(X_{o}, \eta^{*} Y_{o}\right)$ is nonnegative and is in $\mathcal{T}_{C R S-T O}$, provided $\left(X_{o}, Y_{o}\right)$ is nonnegative. In program (5) the factor $\theta$ is minimized and may theoretically become negative. It is straightforward to prove that in such cases the optimal value of the CRS model (5) is unbounded. This is equivalent to the infeasibility of the dual multiplier model (3) and indicates an error in the formulation of weight restrictions (1). Therefore, the latter need to be revisited.

Under the assumption of VRS, the output and input-oriented models (4) and (5) include the additional normalizing equality

$$
\sum_{j=1}^{N} \lambda_{j}=1 .
$$

\footnotetext{
${ }^{5}$ Examples of trade-offs that correspond to homogeneous and non-homogeneous weight restrictions are discussed in Podinovski (2007a) and (2005). Computational algorithms for DEA models with weight restrictions and production trade-offs are developed in Podinovski (2007b).

${ }^{6}$ An axiomatic definition of this technology is given in Podinovski (2004).
} 
Similar to the above, we observe that both VRS models assess the efficiency of the unit $\left(X_{o}, Y_{o}\right)$ in the VRS technology $\mathcal{T}_{V R S-T O}$ that incorporates production trade-offs (6). The definition of this technology is similar to Definition 1, where we require the additional condition (8) to be true.

Example 1. The shaded area in Figure 1 shows the VRS technology with a single input and single output, generated by two observed units $A$ and $B$. Suppose we have specified two linked weight restrictions, where $v_{1}$ and $u_{1}$ are the input and output weights, respectively:

$$
\begin{aligned}
u_{1}-2 v_{1} & \leq 0 \\
-u_{1}+v_{1} & \leq 0
\end{aligned}
$$

These two weight restrictions are special cases of (1). Comparing the latter with (6), the incorporation of weight restrictions (9) in the VRS multiplier model is equivalent to the incorporation of the following two production trade-offs in the VRS envelopment model:

$$
\begin{aligned}
& \left(P_{1}, Q_{1}\right)=(2,1), \\
& \left(P_{2}, Q_{2}\right)=(-1,-1) .
\end{aligned}
$$

Production trade-offs (10) expand the original VRS technology depicted in Figure 1. According to formula (7) (used together with the normalizing equality (8)), these trade-offs can be applied to any unit in the VRS technology and in any proportion $\pi_{t} \geq 0$ (or any number of times), provided the resulting unit is nonnegative.

For example, consider trade-off (10.1). Starting at unit $A$ in Figure 1, and applying this trade-off once, we add 2 units to the input and simultaneously add 1 unit to the output. This results in unit $D$. Continuing this process and allowing the trade-off to be used in any proportions $\pi_{1} \geq 0$, we add the ray $A L$ to the technology. By the assumption of free disposability of output, the area below this ray is also added to the technology.

Similarly, starting at $A$ and applying trade-off (10.2) once, we obtain unit $K$. Applying the same trade-off in different proportions $\pi_{2} \geq 0$ and observing the nonnegativity of the resulting units, we generate the line $W A$. By free disposability, this automatically adds the area below this line to the expanded technology.

We can also apply trade-offs (10) to any other unit in the (expanded) VRS technology, e.g., to unit $B$. However, in our example this does not add new points to technology. Overall, the technology $\mathcal{T}_{V R S-T O}$ is the area below the line $W A L$ and above the horizontal axis. The new efficient frontier is the line $W A L$.

We finally note that the incorporation of weight restrictions (9) in the multiplier DEA model or, equivalently, the incorporation of the corresponding production trade-offs (10) in the dual envelopment model results in an improved discrimination on efficiency. While in the original VRS model both observed units $A$ and $B$ are efficient, in the model with weight restrictions only $A$ remains efficient and unit $B$ is not. For example, in the output orientation, the efficient target of unit $B$ is unit $K$.

\section{Free and unlimited production}

One of well-known potential problems with weight restrictions is that their incorporation in the multiplier model may result in its infeasibility. This of course means that the weight restrictions need to be reassessed. Recently, Podinovski and Bouzdine-Chameeva (2013) linked this problem to the existence of free or unlimited production in the technology expanded by the trade-offs (6).

Let $Y_{o} \in \mathbb{R}_{+}^{s}, Y_{o} \neq 0$, be a vector of outputs. 


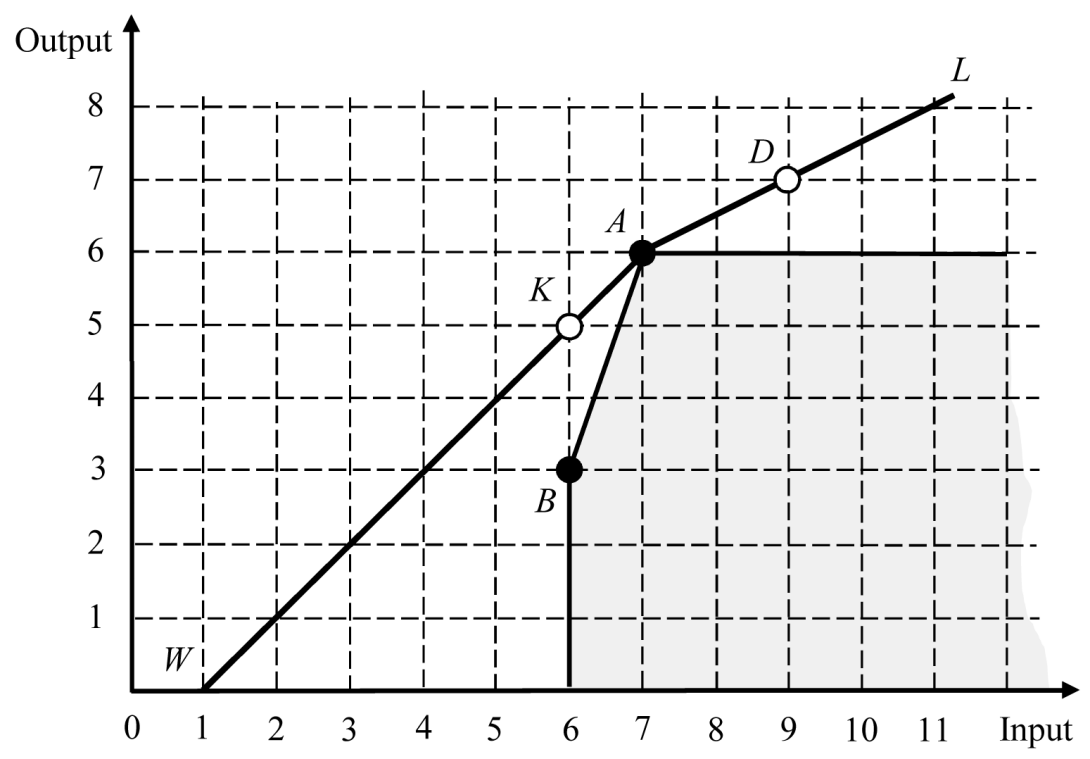

Figure 1: Technology $\mathcal{T}_{V R S-T O}$ defined by units $A$ and $B$, and two linked weight restrictions.

Definition 2. Technology $\mathcal{T}$ allows free production of vector $Y_{o}$ if $\left(X_{o}, Y_{o}\right) \in \mathcal{T}$, where $X_{o}=0$.

Definition 3. Technology $\mathcal{T}$ allows unlimited production of vector $Y_{o}$ if there exists a vector of inputs $X_{o}$ such that $\left(X_{o}, \alpha Y_{o}\right) \in \mathcal{T}$, for all $\alpha \geq 0$.

The free and unlimited production is not allowed by established definitions of production technology (Färe et al. 1985). In the case of technologies $\mathcal{T}_{C R S-T O}$ and $\mathcal{T}_{V R S-T O}$, the existence of free or unlimited production indicates that we made an error in the construction or assessment of weight restrictions. ${ }^{7}$

Podinovski and Bouzdine-Chameeva (2013) give several examples illustrating the fact that the incorporation of unlinked and linked weight restrictions may generate free and unlimited production in the CRS and VRS technologies. In some cases this problem is apparent from the normal computations of efficiency, for example, if the multiplier models are infeasible. However, it is also possible that the problem with weight restrictions may not be apparent. Below we give an additional example that illustrates these observations.

Example 2. Consider the VRS technology generated by observed units $A$ and $B$ and shown as the shaded area in Figure 2. This technology is obtained by shifting the VRS technology in Figure 1 to the left by 3 units of input. Let us show that the same weight restrictions (9) that were unproblematic in Example 1, induce free production in the current example.

As in Example 1, weight restrictions (9), or equivalent to them trade-offs (10), generate the lines $A L$ and $A F$ which are added to the technology. ${ }^{8}$ Note that this creates free production: point $F$ corresponds to the production of 2 units of output from zero input.

This example leads to several observations.

First, the same weight restrictions may be unproblematic for one set of observed units (Figure 1) and induce free production for another (Figure 2).

\footnotetext{
${ }^{7}$ In practice, it may also be worth checking whether the inputs and outputs are properly specified. For example, a missing input may cause free production in both technologies $\mathcal{T}_{V R S-T O}$ and $\mathcal{T}_{C R S-T O}$, while in reality there may be none because not all resources (inputs) are accounted for.

${ }^{8}$ The points on the segment $W F$ have a negative input. By the definition of the VRS technology with production trade-offs (Definition 1 with condition (8)) such units are not members of technology $\mathcal{T}_{V R S-T O}$.
} 


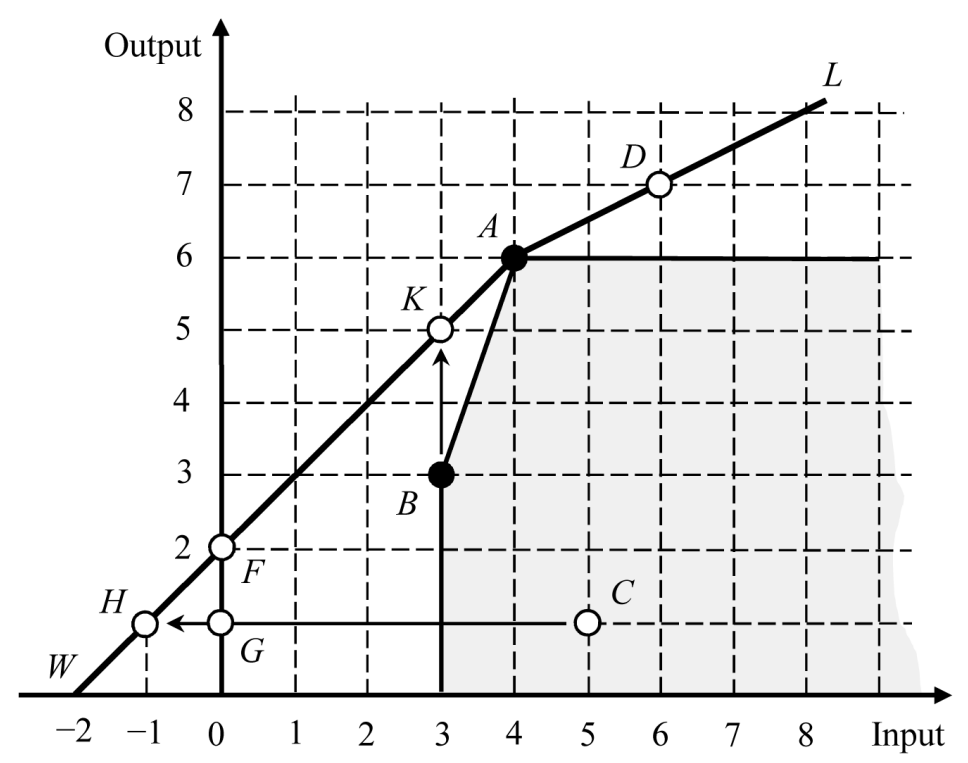

Figure 2: Free production in technology $\mathcal{T}_{V R S-T O}$ with linked weight restrictions.

Second, we may not always be able to observe any problems with the weight restrictions by looking at the efficiency calculations or efficient targets. As an illustration, in technology $\mathcal{T}_{V R S-T O}$ in Figure 2, unit $A$ is efficient. Unit $B$ is inefficient and its efficiency score does not appear problematic. For example, in the output orientation, unit $B$ is projected on $K$ and its output radial efficiency is equal to 0.6 . Yet, the boundary $F A$ is a result of an unacceptable weight restriction (9.2). Therefore, the efficiency of unit $B$ obtained by projection on this boundary is incorrect.

Third, if weight restrictions induce free production, the results of efficiency calculations may be puzzling and meaningless from a practical point of view. To see this, assume that unit $C$ is also observed - this does not affect the technology. Its output radial efficiency is not problematic, although it is understandably very low due to the low level of output at $C$. However, in the input orientation, unit $C$ is projected on the boundary point $G$, and the input radial efficiency of $C$ is 0 . Furthermore, the envelopment model (5) with the additional VRS constraint (8) projects $C$ on the unit $H$, and the resulting input radial "efficiency" is $-1 / 5=-0.2$. (This is because program (5), as the dual to the multiplier model (3), does not specify a nonnegativity condition on $\theta$.) Using the multiplier model instead of the envelopment model would obviously produce the same "efficiency" -0.2 of the unit $C .^{9}$

The notions of free and unlimited production in the CRS and VRS technologies are closely related to each other, which facilitates the testing of whether any given weight restrictions are problematic in this respect. The following two theorems proved in Podinovski and Bouzdine-Chameeva (2013) clarify this issue.

\footnotetext{
${ }^{9}$ It might appear that the problem of negative efficiency $\theta^{*}=-0.2$ may be treated by the incorporation of inequality $\theta \geq 0$ in program (5). Note that this approach would not make the problematic weight restrictions (9) right: the latter would still induce free production in the expanded technology. Furthermore, in the so modified envelopment program the input radial efficiency of $C$ would be equal to 0 (i.e., $\theta^{*}=0$ ), which is meaningless. Furthermore, the modified envelopment program would no longer be dual to the standard multiplier model.
} 
Theorem 1. Technology $\mathcal{T}_{C R S-T O}$ allows free production of vector $Y_{o}$ if and only if it allows its unlimited production.

Note that Theorem 1 does not hold in the VRS technology $\mathcal{T}_{V R S-T O}$. For example, technology $\mathcal{T}_{V R S-T O}$ in Figure 2 allows free production but does not allow unlimited production. ${ }^{10}$

Theorem 2. The existence of free and, therefore, unlimited production of the output vector $Y_{o}$ in technology $\mathcal{T}_{C R S-T O}$ is equivalent to the existence of either free or unlimited production of vector $Y_{o}$ (but not necessarily both) in technology $\mathcal{T}_{V R S-T O}$.

Let us point out two important implications of Theorems 1 and 2.

First, if weight restrictions (1) are problematic (causing free or unlimited production) in either the CRS or VRS model, then the same weight restrictions are problematic in the other model. Reversing this statement, if the weight restrictions do not cause free or unlimited production in one of the two models, then they do not cause a problem in the other.

Second, to see if weight restrictions induce free or unlimited production in either CRS or VRS model of technology, it suffices to test only for the existence of free production in the CRS model.

\section{Consistent weight restrictions}

It is straightforward to verify that, if $X_{j} \neq 0$ for all observed units $j=1, \ldots, N$ (which was assumed above), the standard CRS and VRS technologies do not allow free and unlimited production. Examples in Podinovski and Bouzdine-Chameeva (2013) and the above Example 2 show that the incorporation of weight restrictions may induce free or unlimited production in the expanded technology.

Definition 4. For a given set of observed units $\Omega$, weight restrictions (1) are consistent if the technology $\mathcal{T}_{C R S-T O}$ does not allow free production. Otherwise weight restrictions (1) are inconsistent. (Equivalently, the dual trade-offs (6) are consistent in the former case and inconsistent in the latter.)

Several comments are worth making with regard to the above definition.

- By Theorem 1, weight restrictions (1) are consistent if and only if technology $\mathcal{T}_{C R S-T O}$ does not allow unlimited production. Equivalently, by Theorem 2, weight restrictions (1) are consistent if and only if technology $\mathcal{T}_{V R S-T O}$ does not allow free and unlimited production.

- As Examples 1 and 2 show, the same weight restrictions may be consistent for one set of observed units $\Omega$ and inconsistent for another set $\Omega^{\prime}$. Our main results proved in the next section identify a large class of weight restrictions whose consistency does not depend on the data set. This expands the earlier result of Podinovski and BouzdineChameeva (2013) stating that the consistency of unlinked weight restrictions does not depend on the set of observed units. ${ }^{11}$

\footnotetext{
${ }^{10}$ In Figure 2, the CRS technology (induced by units $A$ and $B$, and trade-offs (10)) is the nonnegative quadrant $\mathbb{R}_{+}^{2}=\{(x, y) \mid x, y \geq 0\}$, where $x$ is the input and $y$ is the output. This technology obviously allows free and unlimited production, for example, of the output $y=2$.

${ }^{11}$ The fact that weight restrictions are consistent irrespective of the data set only means that they do not induce free and unlimited production in the technology. This does not guarantee that the weight restrictions constructed for some data set $\Omega$ remain meaningful in a model based on a different data set $\Omega^{\prime}$. For example, weight restrictions constructed for a data set involving only research-active universities may need adjustment for a set of universities without a strong research agenda, even if the same inputs and outputs are specified.
} 
- If for at least one observed unit $\left(X_{o}, Y_{o}\right)$ the output or input-oriented multiplier model (2) or (3) is infeasible, the weight restrictions (1) are inconsistent. ${ }^{12}$ More generally, the use of inconsistent weight restrictions may also result in strangely-looking and puzzling outcomes of efficiency calculations (as for unit $C$ in Example 2). All such outcomes (generally different for the cases of CRS and VRS) are stated in Theorems 3 and 4 in Podinovski and Bouzdine-Chameeva (2013). Weight restrictions (1) may also be inconsistent even if there are no apparent problems. An illustration to this is Example 2 in which the input and output radial efficiencies of the observed units $A$ and $B$ are unsuspicious finite values.

\section{Conditions for consistent weight restrictions}

Podinovski and Bouzdine-Chameeva (2013) obtained a necessary and sufficient condition for consistent unlinked weight restrictions. Below we extend this condition to include linked weight restrictions that satisfy certain additional assumptions. For this purpose, we restate weight restrictions (1) in the following detailed form:

$$
\begin{aligned}
& Q_{t_{1}}^{\top} u \leq 0, \quad t_{1}=1, \ldots, K_{1}, \\
& -P_{t_{2}}^{\top} v \leq 0, \quad t_{2}=1, \ldots, K_{2}, \\
& Q_{t_{3}}^{\top} u-P_{t_{3}}^{\top} v \leq 0, \quad t_{3}=1, \ldots, K_{3} .
\end{aligned}
$$

We assume that inequalities (11.3) are linked weight restrictions: $P_{t_{3}} \neq 0$ and $Q_{t_{3}} \neq 0$ for each $t_{3}=1, \ldots, K_{3}$.

The practical use of weight restrictions (11) that include linked restrictions, has been demonstrated in different contexts. For example, Thanassoulis et al. (1995) used such weight restrictions in an application of DEA to the provision of perinatal care in England. ${ }^{13}$ Podinovski (2007a), Khalili et al. (2010) and Santos et al. (2011) used this type of weight restrictions in the context of university departments, secondary schools and electricity distribution utilities, respectively.

Theorem 3 (A necessary condition of consistency). Let $\Omega$ be the set of observed units. If the weight restrictions (11) are consistent (for the given $\Omega$ ), then there exist vectors $u^{*}$ and $v^{*}$ that satisfy (11) and the following two conditions:

(i) $u^{*}>0$,

(ii) $v^{*} \geq 0, X_{j}^{\top} v^{*}>0$ for all $j=1, \ldots, N$.

It is worth pointing out that the vectors $u^{*}$ and $v^{*}$ in Theorem 3 and in the results stated below are not required to satisfy any constraints of the multiplier programs (2) or (3), except the weight restrictions (11).

Also note that, if all inputs of all observed units are strictly positive, e.g. $X_{j}>0$ for all $j=1, \ldots, N$, then condition (ii) of Theorem 3 is equivalently stated as $v^{*} \geq 0, v^{*} \neq 0$.

The following result shows that the necessary conditions of Theorem 3 become necessary and sufficient if all components of vectors $P_{t_{3}}, t_{3}=1, \ldots, K_{3}$, are nonnegative.

\footnotetext{
${ }^{12}$ By duality, the infeasibility of the output-oriented multiplier model (2) is equivalent to the unboundness of the objective function of the corresponding envelopment model (4). The latter implies unlimited production of the output vector $Y_{o}$. The infeasibility of the input-oriented multiplier model (3) also indicates either free or unlimited production (Podinovski and Bouzdine-Chameeva 2013).

${ }^{13}$ The linked weight restriction used by Thanassoulis et al. (1995) is an equality. It is equivalently restated as two inequalities (11.3).
} 
Theorem 4 (A necessary and sufficient condition of consistency). Let all vectors $P_{t_{3}} \geq 0$, for all $t_{3}=1, \ldots, K_{3}$, and let $\Omega$ be the set of observed units. Then the weight restrictions (11) are consistent (for the given $\Omega$ ) if and only if there exist vectors $u^{*}$ and $v^{*}$ that satisfy (11) and conditions (i) and (ii) of Theorem 3.

If the linked weight restrictions (11.3) are not specified, the assumption of Theorem 4 that all vectors $P_{t_{3}}$ are nonnegative is removed from the formulation, and the above result becomes Theorem 8 proved in Podinovski and Bouzdine-Chameeva (2013).

Corollary 1. Let $P_{t_{3}} \geq 0$ for all $t_{3}=1, \ldots, K_{3}$. If there exist vectors $u^{*}>0$ and $v^{*}>0$ that satisfy (11), then the weight restrictions (11) are consistent, for any set of observed units $\Omega$.

Corollary 2. Assume that only the linked weight restrictions (11.3) are specified, and the unlinked weight restrictions (11.1) and (11.2) are missing from (11). Let $P_{t_{3}} \geq 0$ for all $t_{3}=1, \ldots, K_{3}$. (Also, as assumed above, $P_{t_{3}} \neq 0$ for all $t_{3}$.) Then the linked weight restrictions (11.3) are consistent, for any set of observed units $\Omega$.

It is worth noting that the validity of assumptions of Corollaries 1 and 2 depends only on the weight restrictions (11) and does not depend on the set of observed units $\Omega$. If such assumptions are true, the weight restrictions are, as stated, consistent for any $\Omega$.

The next result applies to a larger class of weight restrictions (11) than the class identified in Theorem 4. Specifically, it relaxes the assumption that all vectors $P_{t_{3}}$ are nonnegative. It is, however, only a sufficient condition. ${ }^{14}$

Theorem 5 (A sufficient condition of consistency). Let $\Omega$ be the set of observed units. Suppose there exist vectors $u^{*}$ and $v^{*}$ that satisfy the inequalities (11), conditions (i) and (ii) of Theorem 3 and such that $P_{t_{3}}^{\top} v^{*} \geq 0$ for all $t_{3}=1, \ldots, K_{3}$. Then the weight restrictions (11) are consistent (for the given $\Omega$ ).

\section{Examples}

The following examples clarify the application of the theoretical results obtained in the previous section. We use notation $v_{i}$ for input weights and $u_{r}$ for output weights.

Example 3 (Illustration to Theorem 3). Assume there are two inputs and one output. Consider the following two weight restrictions:

$$
v_{1}-v_{2} \leq 0, \quad 2 u_{1}-v_{1}+v_{2} \leq 0 .
$$

It is straightforward to show that the weight restrictions (12) are inconsistent for any set of observed units $\Omega$. Indeed, by adding the two inequalities (12), we obtain

$$
2 u_{1} \leq 0
$$

Therefore, the necessary condition (i) of Theorem 3 cannot be satisfied and the weight restrictions (12) are inconsistent for any $\Omega$.

\footnotetext{
${ }^{14}$ As an illustration, consider weight restrictions (9). As shown in Example 1, these weight restrictions do not induce free or unlimited production in the VRS technology (in Example 1) and therefore (by Theorem 2) in the corresponding CRS technology. However, noting (10.2), $P_{2}=(-1)$, and the product $P_{2}^{\top} v<0$ for any $v>0$. This shows that the conditions of Theorem 5 are not necessary for the absence of free production.
} 
Example 4 (Illustration to Corollary 1). Assume there are two inputs and two outputs. Consider the following three weight restrictions:

$$
-u_{1}+u_{2} \leq 0, \quad v_{1}-v_{2} \leq 0, \quad 2 u_{1}-v_{1} \leq 0
$$

The first two weight restrictions in (13) are unlinked. The third weight restriction is linked and, formally, $Q_{3}=(2,0)^{\top}$ and $P_{3}=(1,0)^{\top}$. Note that vector $P_{3}$ satisfies the nonnegativity assumption $P_{3} \geq 0$ of Corollary 1 . Furthermore, strictly positive weights $u_{1}^{*}=$ $u_{2}^{*}=1$ and $v_{1}^{*}=v_{2}^{*}=2$ satisfy all inequalities (13). By Corollary 1 , weight restrictions (13) are consistent for any set of observed units $\Omega$.

Example 5 (Illustration to Corollary 2). The single weight restriction (9.1) used in Examples 1 and 2 satisfies the conditions of Corollary 2. Therefore, this single weight restriction may be used in the CRS and VRS DEA models and will not induce free or unlimited production, for any $\Omega$.

Example 6 (Illustration to Theorem 5). Assume that the weight restrictions (12) from Example 3 have been modified as follows:

$$
v_{1}-v_{2} \leq 0, \quad 2 u_{1}+v_{1}-v_{2} \leq 0 .
$$

The second of these weight restrictions is linked and the corresponding vector $P=$ $(-1,1)^{\top}$. Note that this does not satisfy the conditions of Theorem 4 and Corollaries 1 and 2. Let us show that all conditions of Theorem 5 are satisfied. Indeed, define $u_{1}^{*}=1$, $v_{1}^{*}=1$ and $v_{2}^{*}=3$. Then the inequalities (14) and both conditions (i) and (ii) of Theorem 3 are satisfied. Moreover,

$$
P^{\top} v^{*}=(-1,1)(1,3)^{\top}=-1+3=2>0 .
$$

By Theorem 5, weight restrictions (14) are consistent for any set of observed units $\Omega$.

Example 7 (Analytical conditions do not apply). Consider the weight restrictions that specify the lower and upper bounds on the ratio of an output to input weight:

$$
a \leq \frac{u_{1}}{v_{1}} \leq b,
$$

where $b \geq a \geq 0$ are some constants. This can be restated as

$$
-u_{1}+a v_{1} \leq 0, \quad u_{1}-b v_{1} \leq 0 .
$$

Note that weight restrictions (9) in Examples 1 and 2 are of this type. The first linked weight restriction in (15) does not satisfy the assumptions of Theorems 4 and $5 .{ }^{15}$ Therefore, these theorems cannot be used to verify if the weight restrictions (15) are consistent. In a practical application, the consistency of such weight restrictions would, as in Examples 1 and 2 , depend on the set of observed units $\Omega$ and could be tested using the computational method of Podinovski and Bouzdine-Chameeva (2013).

Example 8 (Virtual weight restrictions). This type of weight restrictions, introduced by Wong and Beasley (1990), puts the lower and upper limits on the proportion of any virtual

\footnotetext{
${ }^{15}$ For this inequality, $P_{1}=(-a)<0$, which violates the assumption of Theorem 4. Because $P_{1}^{\top} v^{*}=$ $-a v^{*}<0$ for any $v^{*}>0$ (the latter required by condition (ii) of Theorem 3), Theorem 5 does not apply either.
} 
Table 1: University departments.

\begin{tabular}{lllll}
\hline Department & Staff & UG students & Master students & Publications \\
\hline D1 & 45 & 400 & 50 & 39 \\
D2 & 78 & 1500 & 450 & 62 \\
D3 & 55 & 700 & 120 & 26 \\
D4 & 61 & 1000 & 0 & 10 \\
D5 & 89 & 1590 & 180 & 139 \\
\hline
\end{tabular}

input or output in relation to the total virtual input or, respectively, output. For example, let $Y_{j r^{*}}$ be output $r^{*}$ of the unit $j$. Then we can require that the proportion of the virtual output $Y_{j r^{*}} u_{r^{*}}$ be within the range

$$
a_{r^{*}} \leq Y_{j r^{*}} u_{r^{*}} / Y_{j}^{\top} u \leq b_{r^{*}}
$$

where $a_{r^{*}}$ and $b_{r^{*}}$ are constant scalars such that $0 \leq a_{r^{*}} \leq b_{r^{*}} \leq 1$.

After a straightforward linearization, output virtual weight restrictions (16) and their input analogues become unlinked weight restrictions (11.1) and (11.2), respectively. Therefore, the consistency of virtual weight restrictions can be verified by the general results obtained in Section 6 or using the computational methods developed in Podinovski and Bouzdine-Chameeva (2013).

\section{Numerical example}

The following hypothetical example illustrates the results of this paper. Table 1 shows five academic departments from different universities that specialize in the same area of knowledge, e.g., departments of economics. For simplicity, let the single input be staff, and let the three outputs be undergraduate (UG) students, master students and publications per year.

To be specific, consider the output-oriented VRS model. The corresponding efficiency of the five departments is shown in the second column of Table 2.

Consider the following four weight restrictions. These restrictions are constructed using the trade-off approach illustrated in a similar context by Podinovski (2007a).

Trade-off/Weight restriction 1. We assume that any department can reduce the number of master students by 1 and use the released teaching time to increase the number of undergraduate students by 1 , without asking for extra input (staff) and with no detriment to the other outputs (publications).

To state this judgement as a trade-off, we define $P_{1}=(0)$ and $Q_{1}=(1,-1,0)^{\top}$. The corresponding weight restriction is: ${ }^{16}$

$$
u_{1}-u_{2} \leq 0
$$

Trade-off/Weight restriction 2. Similarly, assume that it is technologically feasible for any department to reduce the number of undergraduate students by 2 and instead teach

\footnotetext{
${ }^{16}$ In transforming production trade-offs to weight restrictions, we use the relationship in the general form between trade-offs (6) and weight restrictions (1).
} 
1 master student, without any change to staff and publications. The corresponding trade-off is stated as $P_{2}=(0)$ and $Q_{2}=(-2,1,0)^{\top}$, and is equivalent to the weight restriction

$$
-2 u_{1}+u_{2} \leq 0 .
$$

Trade-off/Weight restriction 3. We now consider a statement that links the input to outputs. For example, suppose we accept that 1 extra member of staff is sufficient to increase the number of undergraduate students by 10 and publications by 0.5 . This statement is represented by the trade-off $P_{3}=(1)$ and $Q_{3}=(10,0,0.5)^{\top}$ and, equivalently, by the linked weight restriction

$$
10 u_{1}+0.5 u_{3}-v_{1} \leq 0
$$

Trade-off/Weight restriction 4. Assume that a reduction of staff by 1 should not lead to the reduction of more that 25 undergraduate students and more than 2 publications per year. The corresponding trade-off is stated as $P_{4}=(-1)$ and $Q_{4}=(-25,0,-2)^{\top}$ and, equivalently, by the linked weight restriction

$$
-25 u_{1}-2 u_{3}+v_{1} \leq 0 .
$$

Before using the above weight restrictions in the calculations of efficiency, let us check if they are consistent with our data set.

If we want to use only the first three weight restrictions, their consistency follows from Corollary 1. Indeed, in this case (19) is the only weight restriction among the three that is linked, and the corresponding vector $P_{3}=(1) \geq 0$, as required by Corollary 1 . Furthermore, all three inequalities (17)-(19) are simultaneously satisfied if, for example, we let

$$
u_{1}^{*}=u_{2}^{*}=u_{3}^{*}=1, v_{1}^{*}=11 .
$$

Therefore, these weight restrictions are consistent. The third column of Table 2 shows the output radial efficiency of the departments in the VRS model that incorporates weight restrictions (17)-(19).

Obviously, any subset of the three weight restrictions is also consistent with the data set. ${ }^{17}$ For example, we may use only the first two weight restrictions instead of all three.

Now consider all four weight restrictions (17)-(20) together. Because $P_{4}=(-1)$, the conditions of Theorems 4 and 5, and Corollaries 1 and 2 are not satisfied, and we cannot use these results to check the consistency of the given weight restrictions. Note that the conditions of Theorem 3 are satisfied, for example by the weights (21), but this is only a necessary condition that does not imply the consistency of the four weight restrictions. ${ }^{18}$

The incorporation of weight restriction (20) alone or together with weight restrictions (17)(19) does not allow us to use the analytical criteria developed in this paper. In this case we can use either of the two computational methods developed in Podinovski and BouzdineChameeva (2013). One of these requires the solution of the linear program (B.1) shown in Appendix B. It has a finite optimal value which proves that the four weight restrictions (17)(20) are consistent with the set of observed departments.

The last column of Table 2 shows the output radial efficiency of all departments in the VRS model with the four weight restrictions (17)-(20).

\footnotetext{
${ }^{17}$ It is clear that Corollary 1 is satisfied for any subset of the three weight restrictions (17)-(19). As an alternative proof, we note that an omission of any weight restriction removes the corresponding trade-off in the definition of technology and makes the latter generally smaller.

${ }^{18}$ As demonstrated in Example 3, the necessary condition of Theorem 3 is useful for the proof that weight restrictions are inconsistent.
} 
Table 2: Output radial efficiency in different VRS models.

\begin{tabular}{llll}
\hline & \multicolumn{3}{c}{ Weight restrictions } \\
\cline { 2 - 4 } Department & None & $(17)-(19)$ & $(17)-(20)$ \\
\hline D1 & 1 & 1 & 0.76 \\
D2 & 1 & 1 & 1 \\
D3 & 0.94 & 0.91 & 0.69 \\
D4 & 1 & 0.85 & 0.66 \\
D5 & 1 & 1 & 1 \\
\hline
\end{tabular}

Table 3: Efficiency with the inconsistent set of weight restrictions (17), (19), (20) and (22).

\begin{tabular}{lllll}
\hline \multirow{2}{*}{ Department } & \multicolumn{2}{c}{ VRS } & \multicolumn{2}{c}{ CRS } \\
\cline { 2 - 5 } & $\begin{array}{l}\text { Output } \\
\text { oriented }\end{array}$ & $\begin{array}{l}\text { Input } \\
\text { oriented }\end{array}$ & $\begin{array}{l}\text { Output } \\
\text { oriented }\end{array}$ & $\begin{array}{l}\text { Input } \\
\text { oriented }\end{array}$ \\
\hline D1 & 0.76 & 0.87 & 0.55 & 0.55 \\
D2 & 0.53 & 0.65 & 0.51 & 0.51 \\
D3 & 0.37 & 0.59 & 0.3 & 0.3 \\
D4 & 0.12 & 0.4 & 0.1 & 0.1 \\
D5 & 1 & 1 & 1 & 1 \\
\hline
\end{tabular}

Now let us show how inconsistent weight restrictions may arise and be tested in the given context.

Example 9. Suppose that we made a mistake and, instead of weight restriction (18), stated the following inequality:

$$
-u_{1}+2 u_{2} \leq 0
$$

The above corresponds to the trade-offs $\hat{P}_{2}=(0)$ and $\hat{Q}_{2}=(-1,2,0)^{\top}$.

Table 3 shows the "efficiency" of the five departments in the output and input-oriented VRS and CRS DEA models. Some of these scores appear low but otherwise not particularly problematic. Note that, as expected, the computation results with the CRS model are identical in the output and input orientations.

It is straightforward to verify that the use of weight restriction (22) instead of (18) creates an inconsistent set of weight restrictions. Indeed, the four weight restrictions (17), (19), (20) and (22) do not satisfy the necessary condition of consistency stated in Theorem 3. To prove this, we add the inequalities (17) and (22) and obtain $u_{2} \leq 0$. The latter contradicts statement (i) of Theorem 3. Alternatively, we can use the computational approach based on a model similar to (B.1) shown in Appendix B to establish the same result.

Example 10. In the above example the origin of inconsistency is obvious: the weight restriction (22) is inconsistent with (17). The following example is more subtle. Suppose we believe the judgement on which the weight restriction (20) is based is too relaxed. More precisely, if staff is reduced by 1 , the number of students should be reduced by no more 
Table 4: Efficiency and solver diagnostic for inconsistent set of weight restrictions (17)-(19) and (23).

\begin{tabular}{lllll}
\hline \multirow{2}{*}{ Department } & \multicolumn{2}{c}{ VRS } & \multicolumn{2}{c}{ CRS } \\
\cline { 2 - 5 } & $\begin{array}{l}\text { Output } \\
\text { oriented }\end{array}$ & $\begin{array}{l}\text { Input } \\
\text { oriented }\end{array}$ & $\begin{array}{l}\text { Output } \\
\text { oriented }\end{array}$ & $\begin{array}{l}\text { Input } \\
\text { oriented }\end{array}$ \\
\hline D1 & 0.53 & 0.51 & unbounded & 0 \\
D2 & 1 & 1 & unbounded & 0 \\
D3 & 0.59 & 0.59 & unbounded & 0 \\
D4 & 0.62 & 0.57 & unbounded & 0 \\
D5 & 1 & 1 & unbounded & 0 \\
\hline
\end{tabular}

than 20 , and publications by more than 1.5 per year. ${ }^{19}$ Therefore, we change (22) to the following weight restriction:

$$
-20 u_{1}-1.5 u_{3}+v_{1} \leq 0
$$

The above corresponds to the trade-off expressed as $\hat{P}_{4}=(-1)$ and $\hat{Q}_{4}=(-20,0,-1.5)^{\top}$.

The incorporation of weight restrictions (17)-(19) and (23) in the VRS model does not signal any obvious problems in either the input or output orientations. Table 4 shows the corresponding efficiency scores. However, the calculations by the output-oriented CRS model result in the unbounded objective function for all departments. By Definition 3 this means the technology allows unlimited production and the weight restrictions are inconsistent. Obviously, the computational test outlined in Appendix B also reveals the same result.

It is interesting to note that the two linked weight restrictions (19) and (23) used without (17) and (18) are consistent with the set of observed departments in Table 1. However, as demonstrated above, the set of all four weight restrictions is inconsistent.

Finally, the same four weight restrictions (17)-(19) and (23) that are inconsistent with the data set in Table 1 become consistent if we change the number of staff of department D5 from 89 to 90 or more. This shows that the consistency of linked weight restrictions (11.3) where the vector $P_{t_{3}}$ has negative components, as in restriction (23), depends on the data set of observed units.

\section{Conclusion}

In a recent paper, Podinovski and Bouzdine-Chameeva (2013) related the infeasibility of multiplier DEA models with weight restrictions to the fact that the latter induce free or unlimited production in the dual envelopment model. Furthermore, it was shown that the infeasibility is only one of several possible outcomes of problematic weight restrictions. In some cases the fact that weight restrictions cause free or unlimited production may not be obvious from the standard efficiency computations.

If weight restrictions induce free or unlimited production (we call such weight restrictions inconsistent), they need to be reassessed. It is therefore important to be able to test whether the CRS or VRS technology with weight restrictions allows free or unlimited production.

\footnotetext{
${ }^{19}$ This view may be supported by the fact that the highest student-to-staff ratio is that of department D2 which is just over 19, and that the highest publication ratio of all departments is also well below 1.5, except department D5 whose publication ratio is 1.56 .
} 
Podinovski and Bouzdine-Chameeva (2013) developed two approaches, computational and analytical, for this purpose. The latter applies only to unlinked weight restrictions, i.e., those that include only input or output weights in each inequality.

In this paper we extended the above analytical approach to the case that includes linked weight restrictions. We formulated necessary and sufficient conditions for the consistency of such weight restrictions and illustrated their use by examples. Outside this development are left linked weight restrictions whose consistency depends on the particular data set of observed units. The consistency of such weight restrictions can be verified using the known computational approach.

The literature on DEA suggests that weight restrictions are predominantly used in the standard CRS and VRS models that aim at the assessment of the input and output radial efficiency. For this reason our results are presented in terms of these two models. However, all these results extend in a straightforward way, without any modification, to many other DEA models that are based on the same standard CRS and VRS technologies. Examples include models based on directional distance function approach of Chambers et al. (1998), DEA models for the assessment of cost and revenue efficiency, and additive DEA model and its variations (see, for example, Thanassoulis et al. 2008).

All such models employ the same CRS or VRS technology and differ only in the way the units are projected on its boundary. Furthermore, the incorporation of weight restrictions (1) in the multiplier form of any of these models induces trade-offs (6) in the dual envelopment form, exactly as in the standard CRS and VRS models considered in our paper. Therefore, weight restrictions (1) and the corresponding trade-offs (6) are consistent in any of these models (i.e., do not induce free and unlimited production in the technology) if and only if they are consistent in the standard CRS and VRS models. This means that all analytical and computational procedures developed in our paper, and earlier in Podinovski and BouzdineChameeva (2013), are applicable regardless of which of the above models is used for the assessment of efficiency. This observation is of course not surprising: the absence of free production is a characteristic of technology, which is separate from the way in which the projection of inefficient units on the boundary is performed. 


\section{Appendix A. Proofs}

Lemma 1. Let $\left(X_{o}, Y_{o}\right) \in \mathcal{T}_{C R S-T O}$ and $Y_{o} \neq 0$. Technology $\mathcal{T}_{C R S-T O}$ allows free production of vector $Y_{o}$ if and only if program (2) is infeasible.

Proof of Lemma 1. By Theorem 1, free production of $Y_{o}$ is equivalent to its unlimited production. The latter is equivalent to the unboundness of the optimal value of program (4) and, by duality, to the infeasibility of (2).

Proof of Theorem 3. Let $\Gamma$ be the set of solutions $(u, v)$ to $(11)$ such that $u, v \geq 0$. Note that $(u, v)=(0,0) \in \Gamma$. Therefore, $\Gamma \neq \varnothing$. Assume that there exists no $(u, v) \in \Gamma$ such that both conditions (i) and (ii) are true. We need to prove that $\mathcal{T}_{C R S-T O}$ allows free production. Under the assumption made, at least one of the following statements must be true:

(a) there exists an $r^{*}=1, \ldots, s$ such that, for all $(u, v) \in \Gamma$, the component $u_{r^{*}}=0$;

(b) there exists an observed unit $j^{*}=1, \ldots, N$ such that, for all $(u, v) \in \Gamma, X_{j^{*}}^{\top} v=0$.

Indeed, assume the converse: for each $r=1, \ldots, s$, there exists a solution $\left(u^{(r)}, v^{(r)}\right) \in \Gamma$ such that $u_{r}^{(r)}>0$. Similarly, for each $j=1, \ldots, N$, there exists a $\left(u^{(j)}, v^{(j)}\right) \in \Gamma$ such that $X_{j}^{\top} v^{(j)}>0$. Let $(\tilde{u}, \tilde{v}) \geq 0$ be the simple average of all $s$ solutions $\left(u^{(r)}, v^{(r)}\right)$ and $N$ solutions $\left(u^{(j)}, v^{(j)}\right)$. Because $\Gamma$ is a convex set, $(\tilde{u}, \tilde{v}) \in \Gamma$. Note that $\tilde{u}>0$ and, for every $j=1, \ldots, N$, we have $X_{j}^{\top} \tilde{v}>0$. This contradicts the last assumption. Therefore, either statement (a) or (b), or both, is true.

Let (a) be true. Let us prove that technology $\mathcal{T}_{C R S-T O}$ allows free production of the output vector $Y_{o}$ such that $\left(Y_{o}\right)_{r^{*}}=1$ and $\left(Y_{o}\right)_{r}=0$, if $r \neq r^{*}$. By Lemma 1 it suffices to prove that program (2) is infeasible with $\left(X_{o}, Y_{o}\right)$, where $X_{o}$ is a zero vector. By (a), $u_{r^{*}}=0$ for any $(u, v) \geq 0$ that satisfies weight restrictions (2.4). Then $Y_{o}^{\top} u=0$, and constraint (2.2) cannot be satisfied.

Similarly, let (b) be true. Let us prove that $\mathcal{T}_{C R S-T O}$ allows free production of $Y_{j^{*}}$. By Lemma 1, it suffices to prove that program (2) is infeasible with $\left(X_{o}, Y_{o}\right)$, where $X_{o}$ is a zero vector and $Y_{o}=Y_{j^{*}}$. By constraint (2.3) for $j=j^{*}$, we have $Y_{j^{*}}^{\top} u-X_{j^{*}}^{\top} v \leq 0$. By (b), we have $X_{j^{*}}^{\top} v=0$ and, therefore, $Y_{j^{*}}^{\top} u \leq 0$, for any $(u, v) \geq 0$ that satisfies weight restrictions (2.4). Therefore, it is impossible to satisfy (2.2), where $Y_{o}=Y_{j^{*}}$, and program (2) is infeasible.

Proof of Theorem 4. Taking into account Theorem 3, we need to prove the sufficiency of conditions (i) and (ii). Consider any unit $\left(0, Y_{o}\right)$, where $Y_{o} \geq 0, Y_{o} \neq 0$. We need to prove that $\left(0, Y_{o}\right) \notin \mathcal{T}_{C R S-T O}$. By Lemma 1 , it suffices to prove that $(2)$ is feasible for $\left(0, Y_{o}\right)$.

Indeed, by condition (i), $u^{*}>0$. Therefore, $Y_{o}^{\top} u^{*}>0$ and there exists an $\alpha>0$ such that $Y_{o}^{\top}\left(\alpha u^{*}\right)=1$. (More precisely, $\alpha=1 /\left(Y_{o}^{\top} u^{*}\right)>0$.) By condition (ii), there exists a sufficiently large $\beta>0$ such that $Y_{j}^{\top}\left(\alpha u^{*}\right)-X_{j}^{\top}\left(\beta v^{*}\right) \leq 0, \forall j=1, \ldots, N$. Therefore, the vectors of input and output weights defined as

$$
u=\alpha u^{*}, \quad v=\beta v^{*}
$$

satisfy constraints (2.2) and (2.3). The weight restrictions (11.1) and (11.2) are also satisfied. It remains to be proved that, by choosing a sufficiently large $\beta>0$, we can satisfy conditions (11.3). Consider any $t_{3}=1, \ldots, K_{3}$. Because $P_{t_{3}}^{\top} \geq 0$, two possibilities arise.

If $P_{t_{3}}^{\top} v^{*}=0$ then, because $\left(u^{*}, v^{*}\right)$ satisfies (11.3), $Q_{t_{3}}^{\top} u^{*} \leq 0$. Therefore, $P_{t_{3}}^{\top}\left(\alpha v^{*}\right)=0$, $Q_{t_{3}}^{\top}\left(\beta u^{*}\right) \leq 0$, and the weights $(u, v)$ defined by (A.1) satisfy (11.3).

If $P_{t_{3}}^{\top} v^{*}>0$, the weights $(u, v)$ defined by (A.1) satisfy (11.3) for all sufficiently large $\beta>0$.

We have proved that the pair $(u, v)$ defined by (A.1), where $\beta>0$ is sufficiently large, satisfies all constraints of program (2), and the latter is feasible. By Lemma $1, \mathcal{T}_{C R S-T O}$ does not allow free production. 
Proof of Corollary 1. Because $v^{*}>0$ and $X_{j} \geq 0, X_{j} \neq 0, \forall j$, condition (ii) of Theorem 4 is true.

Proof of Corollary 2. All conditions of Theorem 4 are satisfied if we take any $u^{*}, v^{*}>0$ where the components of $v^{*}$ are so large that the weight restrictions (11.3) are satisfied. The latter is possible because $P_{t_{3}} \geq 0$ and $P_{t_{3}} \neq 0, \forall t_{3}$.

Proof of Theorem 5. The proof is similar to the first part of the proof of Theorem 4. For all sufficiently large $\beta>0$, the vectors $u$ and $v$ defined by (A.1) satisfy conditions (2.2), (2.3) and weight restrictions (11.1) and (11.2). For each $t_{3}=1, \ldots, K_{3}$, we have two possible cases: $P_{t_{3}}^{\top} v^{*}=0$ and $P_{t_{3}}^{\top} v^{*}>0$. As proved in Theorem 4 , both cases imply that, for sufficiently large $\beta>0$, the vectors $u$ and $v$ defined by (A.1) satisfy the linked weight restriction (11.3).

\section{Appendix B. Examples of models}

In Section 8 we discuss the assessment of the output radial efficiency of five university departments using the VRS model with weight restrictions (17)-(20). Equivalently, we can solve the dual envelopment models with the corresponding production trade-offs.

The following envelopment VRS model is used to assess the output radial efficiency of department D1. Its efficiency is reciprocal to the optimum value $\eta^{*}$ in the following program:

$$
\begin{aligned}
& \eta^{*}=\max \eta \\
& \text { subject to } \\
& 45 \lambda_{1}+78 \lambda_{2}+55 \lambda_{3}+61 \lambda_{4}+89 \lambda_{5}+1 \pi_{3}-1 \pi_{4} \leq 45 \\
& 400 \lambda_{1}+1500 \lambda_{2}+700 \lambda_{3}+1000 \lambda_{4}+1590 \lambda_{5}+1 \pi_{1}-2 \pi_{2}+10 \pi_{3}-25 \pi_{4} \geq 400 \eta \\
& 50 \lambda_{1}+450 \lambda_{2}+120 \lambda_{3}+180 \lambda_{5}-1 \pi_{1}+1 \pi_{2} \geq 50 \eta \\
& 39 \lambda_{1}+62 \lambda_{2}+26 \lambda_{3}+10 \lambda_{4}+139 \lambda_{5}+0.5 \pi_{3}-2 \pi_{4} \geq 39 \eta \\
& \lambda_{1}+\lambda_{2}+\lambda_{3}+\lambda_{4}+\lambda_{5}=1, \\
& \lambda_{j}, \pi_{k} \geq 0, \forall j, k, \eta \text { sign free. }
\end{aligned}
$$

To check the consistency of the four weight restrictions (17)-(20) with the data set, we use model (12) developed by Podinovski and Bouzdine-Chameeva (2013). This model maximizes the sum of all individual outputs in the CRS technology, where the input is kept constant and equal to the input vector of one of the observed units (we take the input of department D1 for this purpose).

$$
\begin{aligned}
& S^{*}=\max y_{1}+y_{2}+y_{3} \\
& \text { subject to } \\
& 45 \lambda_{1}+78 \lambda_{2}+55 \lambda_{3}+61 \lambda_{4}+89 \lambda_{5}+1 \pi_{3}-1 \pi_{4} \leq 45, \\
& 400 \lambda_{1}+1500 \lambda_{2}+700 \lambda_{3}+1000 \lambda_{4}+1590 \lambda_{5}+1 \pi_{1}-2 \pi_{2}+10 \pi_{3}-25 \pi_{4} \geq y_{1}, \\
& 50 \lambda_{1}+450 \lambda_{2}+120 \lambda_{3}+180 \lambda_{5}-1 \pi_{1}+1 \pi_{2} \geq y_{2}, \\
& 39 \lambda_{1}+62 \lambda_{2}+26 \lambda_{3}+10 \lambda_{4}+139 \lambda_{5}+0.5 \pi_{3}-2 \pi_{4} \geq y_{3}, \\
& \lambda_{j}, \pi_{k} \geq 0, \forall j, k, y_{1}, y_{2}, y_{3} \geq 0 .
\end{aligned}
$$

Calculations show that the above linear program has a finite optimal value. (The actual value $S^{*}=2295.744$ is unimportant for this test.) By Theorem 12 in Podinovski and Bouzdine-Chameeva (2013), weight restrictions (17)-(20) are consistent with the set of departments in Table 1. 


\section{References}

Allen, R., Athanassopoulos, A., Dyson, R.G., Thanassoulis, E. (1997) Weights restrictions and value judgements in data envelopment analysis: Evolution, development and future directions. Annals of Operations Research, 73, 13-34.

Banker, R.D., Charnes, A., Cooper, W.W. (1984) Some models for estimating technical and scale efficiencies in data envelopment analysis. Management Science, 30(9), 1078-1092.

Chambers, R.G., Chung, Y., Färe, R. (1998) Profit, directional distance functions, and Nerlovian efficiency. Journal of Optimization Theory and Applications, 98(2), 351-364.

Charnes, A., Cooper, W.W., Rhodes, E. (1978) Measuring the efficiency of decision making units. European Journal of Operational Research, 2(6), 429-444.

Charnes, A., Cooper, W.W., Wei, Q.L., Huang, Z.M. (1989) Cone ratio data envelopment analysis and multi-objective programming. International Journal of Systems Science, 20(7), 1099-1118.

Cooper, W.W., Seiford, L.M., Tone, K. (2007) Data envelopment analysis. A comprehensive text with models, applications, references and DEA-Solver software. 2nd Edition. New York: Springer Science + Busines Media.

Färe, R., Grosskopf, S., Lovell, C.A.K. (1985) The Measurement of Efficiency of Production. Hingham, MA: Kluwer Academic Publishers.

Khalili, M., Camanho, A.S., Portela, M.C.A.S., Alirezaee, M.R. (2010) The measurement of relative efficiency using data envelopment analysis with assurance regions that link inputs and outputs. European Journal of Operational Research, 203(3), 761-770.

Podinovski, V.V. (2004) Production trade-offs and weight restrictions in data envelopment analysis. Journal of the Operational Research Society, 55(12), 1311-1322.

Podinovski, V.V. (2005) The explicit role of weight bounds in models of data envelopment analysis. Journal of the Operational Research Society, 56(12), 1408-1418.

Podinovski, V.V. (2007a) Improving data envelopment analysis by the use of production trade-offs. Journal of the Operational Research Society, 58(10), 1261-1270.

Podinovski, V.V. (2007b) Computation of efficient targets in DEA models with production trade-offs and weight restrictions. European Journal of Operational Research, 181(2), 586-591.

Podinovski, V.V., Bouzdine-Chameeva, T. (2013) Weight restrictions and free production in data envelopment analysis. Operations Research, 61(2), 426-437.

Roll, Y., Cook, W.D., Golany, B. (1991) Controlling factor weights in data envelopment analysis. IIE Transactions, 23(1), 2-9.

Santos, S.P., Amado, C.A.F., Rosado, J.R. (2011) Formative evaluation of electricity distribution utilities using data envelopment analysis. Journal of the Operational Research Society, 62(7), 1298-1319.

Thanassoulis, E., Boussofiane, A., Dyson, R.G. (1995) Exploring output quality targets in the provision of perinatal care in England using data envelopment analysis. European Journal of Operational Research, 80(3), 588-607.

Thanassoulis, E., Portela, M.C.S., Despić, O. (2008) Data envelopment analysis: The mathematical programming approach to efficiency analysis. In H.O. Fried, C.A.K Lovell, S.S. Schmidt (Eds.), The measurement of productive efficiency and productivity growth (pp. 251-420). New York: Oxford University Press.

Thompson, R.G., Langemeier, L.N., Lee, C.T., Lee, E., Thrall, R.M. (1990) The role of multiplier bounds in efficiency analysis with application to Kansas farming. Journal of Econometrics, 46(1-2), 93-108.

Wong, Y.-H.B., Beasley, J.E. (1990) Restricting weight flexibility in data envelopment analysis. Journal of the Operational Research Society, 41(9), 829-835. 